\title{
Prevention of catheter-related bacteremia in children on hemodialysis: time for action
}

\author{
Constantinos J. Stefanidis
}

Received: 15 June 2009/Accepted: 15 June 2009/Published online: 23 July 2009

(C) IPNA 2009

\begin{abstract}
This editorial commentary discusses the strategies for prevention of catheter-related bacteremia (CRB) in children on hemodialysis, which is associated with high morbidity and the increase of hospital cost. There is evidence that the use of arteriovenous fistulae in children on hemodialysis is associated with lower infection rates. Therefore, the use of catheters in these patients should be decreased by improving arteriovenous fistulae use rates or by increasing peritoneal dialysis patient recruitment. However, despite the wide adoption of such policies, hemodialysis catheters are still being used in a significant number of cases. For these patients, implementation of effective strategies for preventing contamination of the catheter hub should be a priority. The appropriate recording and evaluation of $\mathrm{CRB}$ rates are important for assessing preventive policies. In addition, the successful management of a CRB is essential for preventing recurrence of bacteremia. Recently it was documented in a number of randomized clinical trials that antimicrobial lock solutions were effective for preventing CRB. It is suggested that the use of antimicrobial locks should be considered in children who are at high risk of developing CRB, with caution for their long-term use, because of the possibility of bacterial resistance. Now is the time for action, and all preventive steps should be performed simultaneously to minimize the risk of CRB.
\end{abstract}

Keywords Hemodialysis · Children · Vascular access · Catheter-related bacteremia $\cdot$ Intraluminal antimicrobial lock

C. J. Stefanidis $(\bowtie)$

Department of Pediatric Nephrology,

"P. \& A. Kyriakou" Children's Hospital,

Levadias and Thivon Str, Goudi,

111527 Athens, Greece

e-mail: stefanid@hol.gr

\section{Introduction}

Creation and preservation of an adequate vascular hemodialysis (HD) access is a critical issue for children with endstage renal disease (ESRD). Although an arteriovenous fistula (AVF) is preferred for long-term hemodialysis (HD) [1], tunnelled cuffed catheters (TCC) are used more frequently [2]. Actually, TCCs are often the only form of HD access in young children with relatively small vasculature, as the construction of an AVF or placement of an arteriovenous graft (AVG) might be very difficult or even impossible. In addition, TCCs have advantages such as the relative ease of insertion, pain-free dialysis, and immediate use. Catheter-related bacteremia (CRB) has been a major complication of the use of TCCs since their introduction in the early 1980s [3]. CRBs are associated with complications that affect dialysis adequacy, patient quality of life, and the cost of health care. In this issue of Pediatric Nephrology, Onder et al. report the effectiveness of tissue plasminogen activator (TPA) and tobramycin (TPA/TB) for prophylaxis of CRB in high-risk children on chronic hemodialysis [4]. The results of this retrospective study are encouraging, as TPA/TB locks may effectively reduce the rate of $\mathrm{CRB}$ in these patients and prolong the usability of their TCC [4].

TCC in children compared with AVF/AVG have been associated with an almost five-fold increase in hospitalization as a result of infections [5]. The high TCC use in children is partially explained by the expected expeditious transplantation and technical problems of AVF/AVG placement. Fortunately, high transplantation rates and the increased use of peritoneal dialysis (PD) in many countries limits the need for long-term HD in a number of pediatric patients. However, a significant percentage of children still need HD for extended periods of time. This is especially 
true for children who develop peritoneal membrane failure or are at high risk of recurrent disease, or with patients who resume dialysis after a failed renal transplant and are highly sensitized, or in difficult social situations. These children are at high risk to develop CRB, as the duration of TCC use is one of the most important risk factors for bacteremia [6]. Therefore, for such patients it is crucial to prevent CRB and to preserve vascular sites for use later in life. This commentary highlights the issues that should be considered in order to establish the appropriate strategies for preventing CRBs.

\section{Epidemiological facts}

TCCs continue to be the most commonly used vascular access for chronic HD in the pediatric age group, with a usage of $77 \%$ compared with $11 \%$ AVFs and $12 \%$ AVGs, as reported in the North American Pediatric Renal Transplant Cooperative Study (NAPRTCS) [7]. In addition, the cumulative incidence of infection-related hospitalization of pediatric dialysis patients was $40 \%$ in $\mathrm{HD}$ and $51 \%$ in PD [8]. The most important causes of bacteremia were vascular access for hemodialysis patients and peritoneal catheter for patients on PD.

It is encouraging that the incidence of intravasculardevice-associated CRBs has decreased by nearly $40 \%$ during the past decade [9]. This was mainly the result of more consistent implementation of preventative measures. However, the incidence of CRBs still remains high and is a major cause of morbidity among children on HD. Although the true incidence of CRB associated with TCC is not known, an infection rate ranging from 1.6-5.5 episodes 1,000 days has been reported in most studies [10-12]. This broad range may reflect the variations in practice patterns. Undoubtedly, there are many centers not measuring this rate, accepting $\mathrm{CRB}$ as an unpreventable complication. Obviously, appropriate documentation of CRBs and surveillance of bacteremia are critical to infection control in dialysis units and improving quality of care [13]. A Centers for Disease Control and Prevention (CDC) survey of selfreporting US dialysis centers revealed a mean CRB rate of $1.5 / 1,000$ days [14]. Therefore, rates above $2 / 1,000$ days suggest problems with CRB prevention [15].

\section{Pathogenesis of catheter-related bacteremia}

The pathogenesis of CRBs is complex. Both bacterial pathogenicity and biofilm formation play a key role. However, other factors - including catheter surface characteristics, thrombosis, and immunological response-are equally important. For a microorganism to cause CRB, it must first gain access to the extraluminal or intraluminal surface of the TCCs by one of two primary routes, depending on the length of time following the procedure of the catheter placement. Within the first month after TCC placement, catheters are colonized by the external route from the percutaneous tract mainly by organisms from the patient's skin and the hands of the medical personnel [16]. This is very rare in patients whose TCC was appropriately inserted in the operation room. Evidence-based insertion practices that use maximal barrier protection, topical chlorhexidine for skin disinfection before catheter insertion, and postinsertion skin disinfection procedures should be used in all patients to prevent catheter contamination. After the first month, TCCs are usually infected by the internal routes from contamination of the catheter hub, especially when TCC is inserted over a percutaneous guidewire or when it is later manipulated. This is the predominant mode of invasion of TCCs, and appropriate strategies for its preventions should be applied [16]. More rarely, hematogenous contamination of the catheter may occur from remote sources of local infection, such as pneumonia or, more commonly, a new reinserted catheter in patients with inadequately treated CRB might be contaminated hematogenously [17].

The polymer material of TCCs and its minute surface irregularities seem to activate an inflammatory cascade and have a thrombogenic effect [18]. Thrombosis is mainly responsible for the malfunction of TCCs and enhances the adherence and colonization of TCC with microorganisms. These pathogens are usually common environmental microorganisms including gram-positive, gram-negative and Candida. The invading microorganisms adhere to a multilayered complex structure referred to as biofilm [19].

\section{The role of the biofilm}

Electron micrographs of TCCs have revealed complex communities of different microorganisms covered by an extensive matrix, the biofilm [20]. Bacterial biofilms are responsible for the resistance to most antimicrobial treatments in a high number of patients with device-related and other chronic infections, such as otitis media, cholesteatoma, tonsillitis, cystic fibrosis, and prostatitis [21]. Biofilm formation in TCCs begins soon after TCC placement [16] and is the major source of CRB. The responsible bacteria for CRBs initially grow only in biofilms [22] and are recalcitrant to antibiotic therapy and insensitive to host defense mechanisms [23]. It is of interest that usually it is impossible to recover any bacteria from biofilms by traditional cultures. This leads to the misconception that bacteria are totally eradicated after the appropriate antibiotic treatment of a CRB. 
Recently, with the application of modern molecular diagnostics, it was demonstrated unequivocally that bacteria are present in biofilms and are also metabolically active [21]. This might explain why a previous history of CRB remains the most important risk factor for bacteremia. In addition, the microorganisms isolated during CRB recurrence are usually different from those of the initial CRB. This is possibly the result of inadequate treatment of the polymicrobial biofilm, as once the dominant microorganism is eliminated by specific treatment, the suppressed colonies take over [24].

Microorganisms eventually migrate from the biofilm into the bloodstream with the potential to lodge in distant sites [20]. The majority of CRB episodes are relatively uncomplicated, but approximately $10 \%$ of patients with CRB are hospitalized with either severe sepsis (hemodynamic instability or high fever with shaking chills) or a metastatic infection such as endocarditis, osteomyelitis, septic arthritis, or epidural abscess [25]. These complications are 3.5 times more likely to occur when the infection is due to Staphylococcus aureus [26].

It has been recently documented that biofilms on the surfaces of TCCs may grow slowly, and the released circulating planktonic (free-floating) bacteria may be killed by normal defense mechanisms. Therefore, in such cases, the biofilms are essentially nonpathogenic but still responsible for causing inflammation and bacterial dissemination if they increase or if the host becomes compromised. Some factors may predispose dialysis patients to infection. The immunological system might be deficient in some patients with ESRD as a consequence of the accumulation of uremic toxins, neutrophil dysfunction, and hyperparathyroidism [27]. In addition, hypoalbuminemia increases the likelihood of a recurrence of bacteremia among patients treated for an initial CRB [28].

Biofilm is the source of chronic, subclinical inflammation due to the repeated stimulation of monocytes/macrophages. The release of proinflammatory cytokines may not only provide a strong stimulus for a sustained acute phase response, increased plasma levels of C-reactive protein, and erythropoietin resistance, but they may also favor activation of the coagulation pathways, leading to vascular access thrombosis [29]. However, at present, the extent and incidence of biofilm formation in HD patients have not been adequately investigated, as there are no methods to detect biofilm in vivo.

\section{Time for agreement on diagnostic criteria of catheter-related bacteremia}

CRB should be suspected when a child on HD develops fever or chills and there is no evidence for a source of infection after the appropriate clinical and laboratory evaluation. A definitive diagnosis of $\mathrm{CRB}$ can only be made when blood cultures obtained from both the TCC and a peripheral vein grow the same organism. In addition, the Infectious Diseases Society of America has proposed that the colony count of the TCC blood culture should be more than five-fold greater than that of the peripheral vein [30], as a positive blood culture only from the TCC may simply represent colonization [25]. It was shown in oncology patients that a positive blood culture drawn through an indwelling central venous catheter with no confirmation from a culture from a peripheral vein had low positive predictive value. Therefore, a positive result from a catheter needs clinical interpretation and may require confirmation [31], and the diagnosis of CRB in a patient with a positive blood culture only from the TCC should be considered as probable and not definite [25]. However, using the most rigorous definition, it is possible to miss some cases of true CRB. In clinical practice, it is essential to start intravenous antibiotics immediately after obtaining the appropriate blood cultures in children on HD who develop fever or chills with no evidence for a source of infection.

\section{Time for preventing recurrence of catheter-related bacteremia}

The empiric therapy of CRB should include a glycopeptide antibiotic (vancomycin or teicoplanin) for a possible methicillin-resistant Staphylococcus infection and an aminoglycoside or a broad-spectrum cephalosporin for gramnegative bacteria. Obviously, the treatment should be modified when the pathogen has been identified and sensitivities are available. In addition, it is considered imperative to obtain blood cultures 1 week after completing the antibiotic regimen to document that $\mathrm{CRB}$ has been resolved [25]. However, it has been long recognized that treating CRB with systemic antibiotics alone, without catheter removal, is usually ineffective, as almost two thirds of these patients have a recurrence of CRB soon after discontinuation of antibiotics [32, 33]. Therefore, TCC should be removed with simultaneous insertion of a temporary dialysis catheter and then after a few days, a new TTC should be inserted. Although this approach is effective, it has the disadvantage of multiple procedures and the potential risk of central vein thrombosis, which may preclude future placement of a new dialysis catheter at that site [34].

An alternative strategy is to proceed to catheter removal only in the small percentage of patients $(10-15 \%)$ with persistence of fever after 2 or 3 days of the initiation of therapy with the appropriate antibiotics. For the majority of patients, children with resolution of their fever, it is recommended to have their catheter exchange over a 
guidewire (CEG). This approach was reported to be effective in adult HD patients [33, 35]. CEG offers the advantage of vascular access site preservation, which is very important, especially for children on long-term HD. Onder et al. recently reported that the overall and infectionfree survival times of CEG versus removal and replacement of TTCs are comparable in children on HD with CRB [36]. However, the patients of this study were not randomly allocated, and there was a bias, with the sicker patients having their catheters removed and replaced.

More than 20 years ago, a new method for treating CRB in patients who received total parenteral nutrition [37] was first reported. This technique involves instilling a highly concentrated antimicrobial-anticoagulant solution at the end of a dialysis session which "locks" the catheter when it is not in use [38]. Although antibiotics can kill circulating planktonic microorganisms and treat CRBs, they cannot kill the biofilm pathogens that constitute the source of recurrent infections. Therefore, all efforts should be made to prevent biofilm formation [21]. The purpose of utilizing an intraluminal antimicrobial lock solution (AML) is to either prevent seeding or promote eradication of the microorganisms that colonize the catheter. The antimicrobial concentration used to eradicate microorganisms within the biofilm should be 100-1,000 times greater compared with the required concentrations to eliminate planktonic organisms. Such concentrations cannot usually be reached by systemic antibiotic therapy but might be achieved with the use of AMLs [38].

Capdevila et al. in 1993 first reported the successful treatment of 13 episodes of CRB in HD patients with an AML with heparin and vancomycin in conjunction with systemic antibiotics. None of the patients of these study required catheter removal to cure the CRB [39]. In two subsequent studies, with a vancomycin-gentamicin-heparin lock, $35 \%$ and $38 \%$ of patients, respectively, failed AML therapy and required catheter exchange [40, 41]. In a more recent prospective study, similar results were reported when gentamicin was replaced at the AML solutions with ceftazidime because of reported episodes of gentamicininduced ototoxicity. The likelihood of a clinical cure was $87 \%$ for gram-negative infections, $75 \%$ for S. epidermidis infections, and only $40 \%$ for $S$. aureus infections [42]. In addition, all patients with CRBs caused by Enterococcus had a catheter exchange because in a previous study, a high incidence of secondary Candida infection in such patients treated with AML [43] was found. It was evident from this study that the overall success rate of implementing an antibiotic lock protocol for managing CRB in an individual dialysis program will depend on the relative frequencies of different bacterial pathogens.

Early AML application for optimal management of CRBs is essential. In a recent study by Onder et al., it was reported that AMLs are significantly more effective in clearing CRB in children on HD when used early in infection, diminishing the need for catheter exchange [44]. An AML with tissue plasminogen activator (TPA, $1 \mathrm{mg} / \mathrm{ml}$ ), tobramycin $(5 \mathrm{mg} / \mathrm{ml})$, and vancomycin $(5 \mathrm{mg} / \mathrm{ml})$ was used for 2 weeks following the initial empiric treatment of CRB in children on HD. CRBs were successfully cleared in $63 / 76(83 \%)$ cases. The delay on the catheter exchange did not lead to metastatic infections or any other infectious complications [45].

\section{Time for prophylaxis to prevent catheter-related bacteremia}

Central venous access device contamination remains a persistent problem, even after more than 30 years of widespread clinical use. CRBs affect a number of patients requiring chemotherapy, antibiotic administration, or nutrition. However, the risk of contamination remains greater in HD patients because of frequent connections of the hub to dialysis lines, as colonization of TCCs usually occurs from the hub through their lumen. Therefore, the appropriate implementation of an appropriate technique for TCC handling is essential for reducing CRBs. Dialysis nurses have an important role in this standard TCC care. A significant reduction of CRBs by wrapping the catheter hubs with iodine-saturated gauze for $5 \mathrm{~min}$ before removing the catheter caps has been reported, having the dialysis nurse and patient wear masks during catheter connection and disconnection, and minimizing exposure of the catheter to air [11]. In addition, mandatory handwashing, clean gloves, masks, and use of nonocclusive dressings is recommended [46]. It is recommended that the exit site be cleansed with chlorhexidine $2 \%$ with $70 \%$ alcohol at each dialysis session prior to beginning the treatment. Another important recommendation from the CDC is that TCCs should not be used for any purpose but HD, including lab draws and IV therapy access, except in life-threatening emergencies [47]. Detailed recommendations for preventing CRBs were first published in 1973 [48] and are being updated frequently [49]. However, these recommendations are not universally applied [50]. Therefore, meticulous and continuous efforts should be made to provide appropriate training of dialysis personnel for the implementation of these preventive measures.

a. Preventing contamination of the exit site.

The subcutaneous cuff of TCCs promotes the local development of fibrous tissue, which serves as a mechanical barrier to bacterial invasion from the exit site. This is an important barrier, as it is well documented in two prospective studies that CRBs occurred two to three times 
more frequently in HD patients with nontunneled catheters compared with those with TCCs [51, 52]. However, even in patients with TCCs, external route contamination from the percutaneous tract might occur, especially during the first weeks after their insertion. It has been recently documented in a randomized controlled trial that the risk of such infections can be reduced by topical thrice-weekly application of mupirocin ointment at the exit sites of TCCs. Mupirocintreated patients experienced significantly fewer CRBs and a longer time to first bacteremia. It is also encouraging that mupirocin use was not associated with antimicrobial resistance [53]. Unfortunately, such resistance was described in other studies and might limit the effectiveness of long-term use of mupirocin [54]. In addition, all patients of this study had newly inserted TCCs, and there are doubts whether a similar effect could be documented in patients starting this management after long-term HD. Similar positive results were published by Lok et al. with the application of Polysporin ointment (bacitracin, gramicidin, and polymyxin B) or placebo at the exit site [55]. These studies indicate that the application of antibiotic ointments at the exit sites of TCCs might be significant for reducing S. epidermidis- and S. aureus-related CRBs, especially when it is used immediately after TCC insertion. However, long-term use of this regime has to be looked at with caution because of the possibility of antimicrobial resistance and the increase of infections caused by gram-negative bacteria.

\section{b. Preventing contamination of the catheter hub}

Despite the strict recommendations, contact of the catheter hub with the breath of the patient or dialysis personnel remains a major problem because of the high incidence of nasal colonization by Staphylococcus. It has been reported that nasal mupirocin application in such patients led to eradication of nasal $S$. aureus carriage in $96 \%$ of surveillance cultures and to a four-fold reduction in the incidence of $S$. aureus bacteremia during the 2 years period of the study compared with the historic control period. Once- or thriceweekly maintenance regimens of mupirocin were equally efficacious. In addition, the incidence of bacteremia caused by other microorganisms was not significantly affected [56]. Unfortunately there is a relatively high recurrence rate of nasal colonization, and continuous treatment is necessary. Additionally, there is inadequate evidence for the effect of eliminating nasal colonization on the incidence of catheter infection [15]. The use of a mask by the nurse and the patient during TCC connection and disconnection and minimizing exposure of the catheter to air remain the most important interventions to prevent catheter-hub contamination [11].

c. Preventing $\mathrm{CRB}$ with the use of AML

The use of AML in newly inserted TCCs is designed to prevent biofilm formation. Instillation of high-concentration heparin remains the most common method of "locking" a catheter since it was introduced in the early 1970s. There is evidence that heparin stimulates the growth of biofilm in vitro and higher concentrations of heparin increase biofilm faster than lower concentrations [57]. However, a major complication of heparin locks, especially in the perioperative period, is bleeding due to unsuspected anticoagulation [58]. This is the result of leakage of lock solution from the catheter due to parabolic flow patterns in the catheter [59, 60]. Trisodium citrate $(30 \%)$ is an intraluminal locking agent that has been shown to significantly reduce the rate of CRB [61]. However, there is concern that this solution may cause significant hypocalcemia in the cases where higher amount of trisodium citrate accidentally enter systemic circulation. The US Food and Drug Administration (FDA) issued a warning regarding concentrated trisodium citrate after a fatal accident from a mistaken bolus injection of $46.7 \%$ citrate. The FDA also advised that a $4 \%$ citrate solution should be used as an alternative [62]. Recently, it has been shown that a $4 \%$ citrate solution is effective in maintaining catheter patency $[63,64]$ and has not been demonstrated to induce systemic anticoagulation or hypocalcemia. The same study found that the incidence of CRB before versus after switching from heparin to citrate $4 \%$ was similar.

Taurolidine, an antimicrobial agent effective against gram-negative and gram-positive bacteria as well as many types of fungi [65] has been used since the 1970s for a variety of wound types. Recently, it has been added to an AML with a $4 \%$ citrate solution [43]. Betjes and van Agteren evaluated the effect of this AML in patients with newly placed TCCs. Patients of the taurolidine-citrate group had a lower CRB rate compared with patients on heparin lock and had no increased requirement for thrombolytic interventions to maintain catheter patency [66], as was described by Allon [43]. Further studies, possibly with the addition of heparin to AML, might determine whether catheter patency can be improved. Unfortunately, there are no data on the use of this AML in children on HD.

A significant reduction of CRBs was also described with a gentamicin $(40 \mathrm{mg} / \mathrm{ml})$-citrate lock in patients with newly placed TCCs compared with patients on heparin lock. Leakage of gentamicin from this AML was associated with dizziness, deafness, or ataxia in $7.5 \%$ of patients [67]. The risk of ototoxicity is a concern, especially in patients with longterm prophylactic aminoglycoside AML. There has also been one case reported of ototoxicity with the use of an amikacin AML [68]. A similar reduction of CRBs was reported with the use of a lower dose gentamicin $(5 \mathrm{mg} / \mathrm{ml})$ with citrate lock, and there was no evidence of systemic gentamicin exposure [69]. Therefore, the use of this lower gentamicin dose might prevent the development of ototoxicity. 
Recently, 13 studies involving randomized clinical trials evaluated the rate of CRB in patients with AMLs with various antimicrobial agents versus standard heparin locks. AMLs with gentamicin with heparin [69-71] or citrate [67, 72] versus heparin were used in five of the 13 studies. In another two studies, AMLs with gentamicin with heparin were used; however, vancomycin [73] or cefazolin [74] was also added. In the eighth and ninth study, an AML of cefotaxime with heparin was used $[75,76]$. The tenth study compared three different AMLs: gentamicin/citrate, minocycline/ethylenediaminetetraacetic acid (EDTA), and heparin [77]. The 11th study also compared minocycline/EDTA and heparin [78]. Finally, the last two studies compared AMLs of taurolidine/citrate 4\% [43] and citrate 30\% [61] with heparin locks. All these studies documented the effectiveness of AMLs.

Four studies involving meta-analyses of results from these 13 studies were published in 2008. Each metaanalysis evaluated seven to 11 clinical trials, five of which were common in the four studies. In all meta-analysis studies, a beneficial effect of AMLs was found. James et al. reported that the use of intraluminal antibiotics in 11 trials reduced the rate of catheter removal due to complication by $63 \%$ [79]. The meta-analysis of Labriola et al. of nine trials documented that the use of AMLs in TCCs reduced the incidence of CRB by about a factor of three [80]. However, the achieved incidence of CRB in the ALS groups is similar to published reports from units with low CRB incidence (and presumably stricter hygienic measures) [80]. Similarly, the meta-analysis of 11 studies by Yahav et al. showed that the treatment of four patients with AMLs prevented one CRB [81]. However the same meta-is does not support the role of catheter-lock solutions for patients with recurrent CRBs because the effect of AMLs was seen mainly in the first episode of CRB. The meta-analysis of the seven studies of Jaffer et al. found that the CRB rate with AML was 7.7 times less compared with heparin locks, and it was necessary to treat three patients with AML to prevent one CRB per 100 catheter days. In addition, CRB rates in heparin groups were similar in all seven studies (2.1-4.1/1,000 catheter days), and decreases in CRB achieved by using the different ALS were of similar magnitude [82].

The major problems of all trials are the use of cointerventions (i.e. application of antibiotic ointment at the exit site, use of nasal mupirocin) and the potential publication bias. Trials with negative results might not have been published; therefore, a possible selection bias could have occurred. It is encouraging that the recent meta-analysis studies do not provide evidence for the development of resistance with the use of AML [81]. Unfortunately, the limited follow-up of patients included in these studies does not exclude the onset of adverse events or bacterial resistance with longer use of ALS [80]. Actually, there is some evidence that this possibility might occur. Monthly cultures of the bacteria colonizing the catheter lumen were obtained in a French dialysis unit routinely using gentamicin AMLs. After 2 years, $100 \%$ of the S. epidermidis isolates were resistant to most antibiotics [83]. The use of AML with taurolidine, trisodium citrate $(30 \%)$, or EDTA might be of less concern, as bacterial resistance has not been reported to these agents. The possibility of bacterial resistance was the reason that the 2006 US National Kidney Foundation Kidney Disease Outcomes Quality Initiative (K/DOQI) vascular access guidelines did not include a recommendation for prophylactic antibiotics [49]. However, most studies documenting AML effectiveness were published after the development of these guidelines. In contrast, the CDC guidelines state that catheter-lock solutions can be considered only under special circumstances, such as multiple CRBs, despite maximal adherence to aseptic technique [47]. Finally, AML use should be considered in patients who are at high risk of developing CRB. This group of children includes patients with a history of recurrent infections or on immunosuppression, with a femoral TCC, a decreased serum albumin, or an elevated serum ferritin. In addition, AML use should be considered in children who are using one of their last potential dialysis access sites [84].

\section{Conclusions}

Catheter-related bacteremia in children in many hemodialysis centers remains a major concern because of the associated high morbidity and significant increase of hospital cost. Unfortunately, there is still a lack of documentation of the $\mathrm{CRB}$ rate in many centers. Appropriate CRB recording and surveillance should be a priority. A hemodialysis unit with a CRB rate above 2/1,000 days should focus on educating dialysis personnel in the use of appropriate sterile techniques [15].

Another important step for CRB prevention is to decrease the use of TCC, especially in children on long-term dialysis, who are at high risk to develop CRB. Successful AVF placement might be difficult or even impossible, especially in small children. However, numerous studies of successful AVF creation with microsurgical techniques in such patients have been reported. The recommendation of the K/DOQI guidelines for increasing the number of fistulas should be applied also in children. Early referral of children might be very important for enhancing "fistula-first" strategy. An alternative approach is to consider peritoneal dialysis or preemptive transplantation as first choice management in every child with ESRD.

Implementation of appropriate strategies for preventing contamination of the exit site and the catheter hub should 
be a priority. Finally, there is enough evidence to support the prophylactic use of AML in a number of children with increased risk of developing CRB. Now is the time for action, and all the preventive steps should be performed simultaneously in order to minimize the risk of CRB.

\section{References}

1. Fischbach M, Edefonti A, Schröder C, Watson A, The European Pediatric Dialysis Working Group (2005) Hemodialysis in children: general practical guidelines. Pediatr Nephrol 20:10541066

2. Fadrowski JJ, Hwang W, Neu AM, Fivush BA, Furth SL (2009) Patterns of use of vascular catheters for hemodialysis in children in the United States. Am J Kidney Dis 53:91-98

3. Mahan JD Jr, Mauer SM, Nevins TE (1983) The Hickman catheter: a new hemodialysis access derice for infants and small children. Kidney Int 24:694-697

4. Onder AM, Chandar J, Billings AA, Simon N, Gonzalez J, Francoeur D, Abitbol C, Zilleruelo G (2009) Prophylaxis of catheter-related bacteremia using tissue plasminogen activatortobramycin locks. Pediatr Nephrol. doi:10.1007/s00467-0091235-5

5. Fadrowski JJ, Hwang W, Frankenfield DL, Fivush BA, Neu AM, Furth SL (2006) Clinical course associated with vascular access type in a national cohort of adolescents who receive hemodialysis: findings from the Clinical Performance Measures and US Renal Data System projects. Clin J Am Soc Nephrol 1:987-992

6. Lemaire X, Morena M, Leray-Moragués H, Henriet-Viprey D, Chenine L, Defez-Fougeron C, Canaud B (2009) Analysis of risk factors for catheter-related bacteremia in 2000 permanent dual catheters for hemodialysis. Blood Purif 28:21-28

7. North American Pediatric Renal Transplant Cooperative Study (2008) Annual Report. Renal transplantation, dialysis, chronic renal insufficiency. Available at: https://web.emmes.com/study/ ped/annlrept/Annual Report-2008.pdf

8. Chavers BM, Solid CA, Gilbertson DT, Collins AJ (2007) Infection-related hospitalization rates in pediatric versus adult patients with end-stage renal disease in the United States. J Am Soc Nephrol 18:952-959

9. Centers for Disease Control and Prevention (2000) Monitoring hospital acquired infections to promote patient safety-United States, 1990-1999. MMWR Morb Mortal Wkly Rep 49:149-153

10. Saxena AK, Panhorota BR, Al-Mulhim AS (2005) Vascular access related infections in hemodialysis patients. Saudi J Kidney Dis Transplant 16:46-71

11. Beathard GA (2003) Catheter management protocol for catheterrelated bacteremia prophylaxis. Semin Dial 16:403-405

12. Hannah EL, Stevenson KB, Lowder CA, Adcox MJ, Davidson RL, Mallea MC, Narasimhan N, Wagnild JP (2002) Outbreak of hemodialysis vascular access site infections related to malfunctioning permanent tunneled catheters: making the case for active infection surveillance. Infect Control Hosp Epidemiol 23:538-541

13. George A, Tokars JI, Clutterbuck EJ, Bamford KB, Pusey C, Holmes AH (2006) Reducing dialysis associated bacteraemia, and recommendations for surveillance in the United Kingdom: prospective study. BMJ 17 332:1435

14. Klevens RM, Tokars JI, Andrus M (2005) Electronic reporting of infections associated with hemodialysis. Nephrol News Issues 19:37-38

15. Beathard GA, Urbanes A (2008) Infection associated with tunneled hemodialysis catheters. Semin Dial 21:528-538
16. Trautner B, Darouiche R (2004) Catheter-associated infections. Arch Intern Med 164:842-850

17. Crnich CJ, Maki DG (2002) The promise of novel technology for the prevention of intravascular device-related bloodstream infection, I: Pathogenesis and short-term devices. Clin Infect Dis 34:1232-1342

18. Suojanen JN, Brophy DP, Nasser I (2000) Thrombus on indwelling central venous catheters: the histopathology of "fibrin sheaths". Cardiovasc Intervent Radiol 23:194-197

19. Hall-Stoodley L, Costerton JW, Stoodley P (2004) Bacterial biofilms: from the natural environment to infectious diseases. Nat Rev Microbiol 2:95-108

20. Donlan RM (2002) Biofilms microbial life on surfaces. Emerg Infect Dis 9:881-890

21. Costerton W, Veeh R, Shirtliff M, Pasmore M, Post C, Ehrlich G (2003) The application of biofilm science to the study and control of chronic bacterial infections. J Clin Invest 112:1466-1477

22. Marrie TJ, Costerton JW (1984) Scanning and transmission electron microscopy of in situ bacterial colonization of intravenous and intraarterial catheters. J Clin Microbiol 19:687-693

23. Ward KH, Olson ME, Lam K, Costerton JW (1992) Mechanism of persistent infection associated with peritoneal implants. J Med Microbiol 36:406-413

24. Rijnders BJ, Van Wijngaerden E, Van Eldere J, Peetermans WE (2001) Polyclonal Staphylococcus epidermidis intravascular catheter related infections. Clin Microbiol Infect 7:388-391

25. Allon M (2004) Dialysis catheter-related bacteremia: treatment and prophylaxis. Am J Kidney Dis 4:779-791

26. Mokrzycki MH, Zhang M, Cohen H, Golestaneh L, Laut JM, Rosenberg SO (2006) Tunnelled hemodialysis catheter bacteraemia: risk factors for bacteraemia recurrence, infectious complications and mortality. Nephrol Dial Transplant 21:1024-1031

27. Jaber BL (2005) Bacterial infections in hemodialysis patients: pathogenesis and prevention. Kidney Int 67:2508-2519

28. Tanriover B, Carlton D, Saddekni S, Hamrick K, Oser R, Westfall AO, Allon M (2000) Bacteremia associated with tunneled dialysis catheters: comparison of two treatment strategies. Kidney Int 57:2151-2155

29. Canaud B, Sénécal L, Leray-Moragués H, Picard-Gontiers A, Terrier N, Morena M, Cristol JP (2003) Vascular access, an underestimated cause of inflammation in hemodialysis patient. Néphrologie 24:353-358

30. Mermel LA, Farr BM, Sherertz RJ, Issam I, Raad II, O'Grady N, Harris JS, Craven DE (2001) Guidelines for the management of intravascular catheter-related infections. Clin Infect Dis 32:1249-1272

31. DesJardin J (1999) Clinical utility of blood cultures drawn from indwelling central venous catheters in hospitalized patients with cancer. Ann Intern Med 131:641-647

32. Marr KA, Sexton DJ, Conlon PJ, Corey GR, Schwab SJ, Kirkland KB (1997) Catheter-related bacteremia and outcome of attempted catheter salvage in patients undergoing hemodialysis. Ann Intern Med 127:275-280

33. Saad TF (1999) Bacteremia associated with tunneled, cuffed hemodialysis catheters. Am J Kidney Dis 34:1114-1124

34. Allon M (2005) Saving infected catheters: why and how. Blood Purif 23:23-28

35. Robinson D, Suhocki P, Schwab SJ (1998) Treatment of infected tunneled venous access hemodialysis catheters with guidewire exchange. Kidney Int 53:1792-1794

36. Onder AM, Chandar J, Saint-Vil M, Lopez-Mitnik G, Abitbol CL, Zilleruelo G (2007) Catheter survival and comparison of catheter exchange methods in children on hemodialysis. Pediatr Nephrol 22:1355-1361

37. Messing B, Peitra-Cohen S, Beliah M, Bernier J (1988) Antibiotic- lock technique: an approach to optimal therapy for catheter-related sepsis in home parenteral nutrition patients. JPEN J Parenter Enteral Nutr 12(2):185-189 
38. Manierski C, Besarab A (2006) Antimicrobial locks: putting the lock on catheter infections. Adv Chronic Kidney Dis 13:245-258

39. Capdevila JA, Segarra A, Planes AM, Ramírez-Arellano M, Pahissa A, Piera L, Martínez-Vázquez JM (1993) Successful treatment of haemodialysis catheter-related sepsis without catheter removal. Nephrol Dial Transplant 8:231-234

40. Krishnasami Z, Carlton D, Bimbo L, Taylor ME, Balkovetz DF, Barker J, Allon M (2002) Management of hemodialysis catheterrelated bacteremia with an adjunctive antibiotic lock solution. Kidney Int 61:1136-1142

41. Vardhan A, Davies J, Daryanani I, Crowe A, McClelland P (2002) Treatment of haemodialysis catheter-related infections. Nephrol Dial Transplant 17:1149-1150

42. Poole CV, Carolton D, Bimbo L, Allon M (2004) Treatment of catheter-related bacteraemia with an antibiotic lock protocol: effect of bacterial pathogen. Nephrol Dial Transplant 19:12371244

43. Allon M (2003) Prophylaxis against dialysis catheter-related bacteremia with a novel antimicrobial lock solution. Clin Infect Dis 36:1539-1544

44. Onder AM, Chandar J, Billings AA, Simon N, Diaz R, Francoeur D, Abitbol C, Zilleruelo G (2008) Comparison of early versus late use of antibiotic locks in the treatment of catheter-related bacteremia. Clin J Am Soc Nephrol 3:1048-1056

45. Onder AM, Chandar J, Simon N, Saint-Vil M, Francoeur D, Nwobi O, Abitbol C, Zilleruelo G (2008) Treatment of catheterrelated bacteremia with tissue plasminogen activator antibiotic locks. Pediatr Nephrol 23:457-464

46. Mokrzycki MH (2008) Use of prophylactic topical or intraluminal antibiotics for hemodialysis catheters. Nat Clin Pract Nephrol $4: 478-479$

47. O'Grady NP, Alexander M, Dellinger EP, Gerberding JL, Heard SO, Maki DG, Masur H, McCormick RD, Mermel LA, Pearson ML, Raad II, Randolph A, Weinstein RA (2002) Guidelines for the prevention of intravascular catheter-related infections. Centers for Disease Control and Prevention. MMWR Recomm Rep 51:129

48. Maki DG, Goldman DA, Rhame FS (1973) Infection control in intravenous therapy. Ann Intern Med 79:867-887

49. National Kidney Foundation: K/DOQI Guidelines. Clinical practice guidelines for vascular access, 2006 update. Available at www.kidney.org

50. Rubinson L, Wu AW, Haponik EE, Diette GB (2005) Why is it that internists do not follow guidelines for preventing intravascular catheter infections? Infect Control Hosp Epidemiol 26:525-533

51. Stevenson KB, Hannah EL, Lowder CA (2002) Epidemiology of hemodialysis vascular access infections from longitudinal infection surveillance data. Predicting the impact of NKF-DOQI clinical practice guidelines for vascular access. Am J Kidney Dis 39:549-555

52. Weijmer MC, Vervloet MG, ter Wee PM (2004) Compared to tunnelled cuffed haemodialysis catheters, temporary untunnelled catheters are associated with more complications already within 2 weeks of use. Nephrol Dial Transplant 19:670-677

53. Johnson DW, MacGinley R, Kay TD, Hawley CM, Campbell SB, Isbel NM, Hollett P (2002) A randomized controlled trial of topical exit site mupirocin application in patients with tunnelled, cuffed haemodialysis catheters. Nephrol Dial Transplant 17:1802-1807

54. Cookson BD (1990) Mupirocin resistance in staphylococci. J Antimicrob Chemother 25:497-501

55. Lok CE, Stanley KE, Hux JE, Richardson R, Tobe SW, Conly J (2003) Hemodialysis infection prevention with polysporin ointment. J Am Soc Nephrol 13:169-179

56. Boelaert JR, Van Landuyt HW, Godard CA, Daneels RF, Schurgers ML, Matthys EG, De Baere YA, Gheyle DW, Gordts BZ, Herwaldt LA (1993) Nasal mupirocin ointment decreases the incidence of Staphylococcus aureus bacteraemias in haemodialysis patients. Nephrol Dial Transplant 8:235-239

57. Shanks RM, Donegan NP, Graber ML, Buckingham SE, Zegans ME, Cheung AL, O'Toole GA (2005) Heparin stimulates Staphylococcus aureus biofilm formation. Infect Immun 73:4596-4606

58. Moritz ML, Vats A, Ellis D (2003) Systemic anticoagulation and bleeding in children with hemodialysis catheters. Pediatr Nephrol 18:68-70

59. Polaschegg HD, Shah C (2003) Overspill of catheter locking solution: safety and efficacy aspects. ASAIO J 49:713-715

60. Sungur M, Eryuksel E, Yavas S, Bihorac A, Layon AJ, Caruso L (2007) Exit of catheter lock solutions from double lumen acute haemodialysis catheters an in vitro study. Nephrol Dial Transplant 22:3533-3537

61. Weijmer MC, van den Dorpel MA, Van de Ven PJ, ter Wee PM, van Geelen JA, Groeneveld JO, van Jaarsveld BC, Koopmans MG, le Poole CY, Schrander-Van der Meer AM, Siegert CE, Stas KJ, CITRATE Study Group (2005) Randomized, clinical trial comparison of trisodium citrate $30 \%$ and heparin as catheterlocking solution in hemodialysis patients. J Am Soc Nephrol $16: 2769-2777$

62. US Food and Drug Administration (2000) FDA issues warning on tricitrasol dialysis catheter anticoagulant. FDA talk paper TOO16, April 14

63. MacRae JM, Dojcinovic I, Djurdjev O, Jung B, Shalansky S, Levin A, Kiaii M (2008) Citrate 4\% versus Heparin and the Reduction of Thrombosis Study (CHARTS). Clin J Am Soc Nephrol 3:369-374

64. Grudzinski L, Quinan P, Kwok S, Pierratos A (2007) Sodium citrate $4 \%$ locking solution for central venous hemodialysis catheters-an effective, more cost-efficient alternative to heparin. Nephrol Dial Transplant 22:471-476

65. Traub WH, Leonhard B, Bauer D (1993) Taurolidine: in vitro activity against multiple-antibiotic-resistant, nosocomially significant clinical isolates of Staphylococcus aureus, Enterococcus faecium, and diverse Enterobacteriaceae. Chemotherapy 39:322330

66. Betjes MG, van Agteren M (2004) Prevention of dialysis catheterrelated sepsis with a citrate-taurolidine-containing lock solution. Nephrol Dial Transplant 19:1546-1551

67. Dogra $\mathrm{GK}$, Herson $\mathrm{H}$, Hutchison $\mathrm{B}$, Irish $\mathrm{AB}$, Heath $\mathrm{CH}$, Golledge C, Luxton G, Moody H (2002) Prevention of tunneled hemodialysis catheter-related infections using catheter-restricted filling with gentamicin and citrate: a randomized controlled study. J Am Soc Nephrol 13:2133-2139

68. Saxena AK, Panhotra BR, Naguib M (2002) Sudden irreversible sensory-neural hearing loss in a patient with diabetes receiving amikacin as an antibiotic-heparin lock. Pharmacotherapy 22:105108

69. McIntyre CW, Hulme LJ, Taal M (2004) Locking of tunneled hemodialysis catheters with gentamicin and heparin. Kidney Int 66:801-805

70. Cooper R, Saad T (1999) Prevention of bacteraemia in patients with tunnelled cuffed "permanent" hemodialysis catheters (PCs) using gentamicin catheter packing. J Am Soc Nephrol 10:203A

71. Zhang P, Zhang W, He Q, Yuan J, Xie W, Jiang W, Jiang H, Chen $\mathrm{J}$ (2006) A randomized controlled study on prevention of cufftunneled catheter related bacteremia with gentamicin-heparin lock solution: the metaphase result [abstract]. J Am Soc Nephrol 17:592A

72. Pervez A, Ahmed M, Ram S, Torres C, Work J, Zaman F, Abreo K (2002) Antibiotic lock technique for prevention of cuffed tunnel catheter associated bacteremia. J Vasc Access 3:108-113 
73. Al-Hwiesh AK, Abdul-Rahman IS (2007) Successful prevention of tunneled, central catheter infection by antibiotic lock therapy using vancomycin and gentamycin. Saudi J Kidney Dis Transpl $18: 239-247$

74. Kim SH, Song KI, Chang JW (2006) Prevention of uncuffed hemodialysis catheter-related bacteremia using an antibiotic lock technique: a prospective, randomized clinical trial. Kidney Int 69:161-164

75. Saxena AK, Panhotra BR (2005) The impact of catheter-restricted filling with cefotaxime and heparin on the lifespan of temporary hemodialysis catheters: a case controlled study. J Nephrol 18:755763

76. Saxena AK, Panhotra BR, Sundaram DS, Morsy MN, Al-Ghamdi AM (2006) Enhancing the survival of tunneled haemodialysis catheters using an antibiotic lock in the elderly: a randomised, double-blind clinical trial. Nephrology (Carlton) 11:299-305

77. Nori US, Manoharan A, Yee J, Besarab A (2006) Comparison of low-dose gentamicin with minocycline as catheter lock solutions in the prevention of catheter-related bacteremia. Am J Kidney Dis 48:596-605

78. Bleyer AJ, Mason L, Russell G, Raad II, Sherertz RJ (2005) A randomized, controlled trial of a new vascular catheter flush solution (minocycline- EDTA) in temporary hemodialysis access. Infect Control Hosp Epidemiol 26:520-524
79. James MT, Conley J, Tonelli M, Manns BJ, MacRae J, Hemmelgarn BR, Alberta Kidney Disease Network (2008) Meta-analysis: antibiotics for prophylaxis against hemodialysis catheter-related infections. Ann Intern Med 148:596-605

80. Labriola L, Crott R, Jadoul M (2008) Preventing haemodialysis catheter-related bacteraemia with an antimicrobial lock solution: a meta-analysis of prospective randomized trials. Nephrol Dial Transplant 23:1666-1672

81. Yahav D, Rozen-Zvi B, Gafter-Gvili A, Leibovici L, Gafter U, Paul M (2008) Antimicrobial lock solutions for the prevention of infections associated with intravascular catheters in patients undergoing hemodialysis: systematic review and meta-analysis of randomized, controlled trials. Clin Infect Dis 47:83-93

82. Jaffer Y, Selby NM, Taal MW, Fluck RJ, McIntyre CW (2008) A metaanalysis of hemodialysis catheter locking solutions in the prevention of catheter-related infection. Am J Kidney Dis 51:233-241

83. Guerraoui AA, Dacosta EE, Roche BB (2004) Emergence of multiresistant Staphylococcus epidermidis (MRSE) after lock antibiotic regimen by gentamicin in permanent hemodialysis catheters. Prospective study, 1999-2003 [abstract]. J Am Soc Nephrol 15:368A

84. Bleyer AJ (2007) Use of antimicrobial catheter lock solutions to prevent catheter-related bacteremia. Clin J Am Soc Nephrol 2:1073-1078 